\title{
Crystal Growth of Low-Temperature Processed Poly-Si by Excimer Laser Annealing —Dependences of Poly-Si Grain on Energy Density and Shot Number-
}

\author{
Naoto MATSUO, Hisashi ABE*, Naoya KAWAMOTO, Ryouhei TAGUCHI, \\ Tomoyuki NOUDA*, Hiroki HAMADA* and Tadaki MIYOSHI \\ Department of Electrical \& Electronic Engineering, Yamaguchi University, \\ Tokiwadai, Ube 755-8611, Japan.e-mail:nmatsuo@po.cc.yamaguchi-u.ac.jp \\ *Microelectronics Research Center, SANYO Electric Co., Ltd, \\ 180, Ohmori, Anpachi-cho, Anpachi-gun, Gifu 503-0195, Japan
}

(Received July 28, 2000, Accepted October 7, 2000)

\begin{abstract}
This paper examines the characteristics of the poly-Si grains formed by ELA with the energy density from $75 \mathrm{~mJ} /$ $\mathrm{cm}^{2}$ to $400 \mathrm{~mJ} / \mathrm{cm}^{2}$, and discusses the change of the crystal growth mechanism of the recrystallized poly-Si dependent on the energy density. For the energy density from $250 \mathrm{~mJ} / \mathrm{cm}^{2}$ to $350 \mathrm{~mJ} / \mathrm{cm}^{2}$, the disk-shaped grains are observed. The dependences of the area ratio of disk-shaped grain both on the energy density and on the shot number are examined. From these results, the role of the disk-shaped grains for the change of the crystal growth mechanism dependent on the energy density is clarified.
\end{abstract}

Keywords: poly-Si grains, ELA, disk-shaped grains, crystal growth mechanism

\section{Introduction}

The characteristics of the recrystallized poly-Si film prepared by excimer laser annealing (ELA) and that of the thin film transistors (TFTs) fabricated on the film have been examined ${ }^{1,2}$. The growth model of the recrystallized poly-Si under the high energy density has been presented from a viewpoint of the nucleation from the super cooled liquid $(\mathrm{SCL})^{3)}$. However, considering the fact that the entirc irradiated amorphous $\mathrm{Si}$ (a-Si) becomes poly-Si grains after a certain shot number for multi-pulse laser irradiation under a low energy density, smaller than $250 \mathrm{~mJ} / \mathrm{cm}^{2}$ and the melting point of the poly-Si is higher than that of a-Si, the super cooled liquid is not thought to be formed in the range above the shot number. We have examined the characteristics of the low-temperature processed poly-Si formed at the energy density of $75 \sim 250 \mathrm{~mJ} / \mathrm{cm}^{2}$, and presented a new crystal growth model based on the solid phase crystallization (SPC) which proceeds during low-energy density ELA. The crystal growth mechanism is understood through the dislocation movement ${ }^{4}$.

This paper examines the characteristics of the poly-Si grains formed by ELA with the energy density from $75 \mathrm{~mJ} / \mathrm{cm}^{2}$ to $400 \mathrm{~mJ} / \mathrm{cm}^{2}$, and discusses the crystal growth mechanism of the recrystallized
poly-Si. For the energy density from $250 \mathrm{~mJ} / \mathrm{cm}^{2}$ to $350 \mathrm{~mJ} / \mathrm{cm}^{2}$, the disk-shaped grains are observed. First, the dependence of the ratio of diskshaped grain on the energy density is examined. Secondly, the dependence of that of the diskshaped grains on the shot number is examined. From these results, the change of the crystal growth mechanism depending on the energy density and shot number is discussed.

\section{Growth and Analysis Methods}

An a-Si film is deposited on the quartz substrate by the low pressure chemical vapour deposition (LPCVD) method using $\mathrm{Si}_{2} \mathrm{H}_{6}$ for the film with the hydrogen concentration $\left(\mathrm{H}_{2}\right.$ conc.) of $4 \mathrm{E} 20$. The film thickness is $100 \mathrm{~nm}$. The $\mathrm{KrF}$ multi-pulse excimer laser is irradiated after the a-Si formation at $1 \mathrm{~Hz}$ and at room temperature under approximately $10^{-4} \mathrm{~Pa}$. The irradiation time is $23 \mathrm{nS}$ at full width at half maximum (FWHM). The energy density and the shot number are $200-400 \mathrm{~mJ} / \mathrm{cm}^{2}$ and $8-100$, respectively. The crystallinity and surface morphology of the poly-Si film are measured by the Raman spectroscopy and scanning electron microscopy (SEM), respectively. Secco etching was performed before obseving the poly-Si surface by SEM to clarify the film structure and the morphology. 


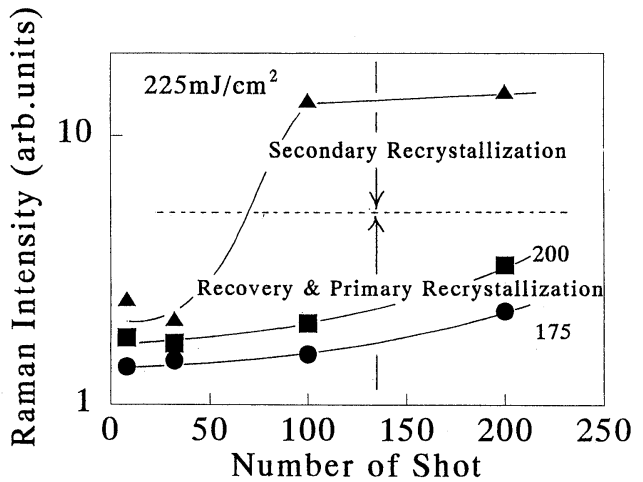

Fig. 1 Relationship between the Raman intensity and shot number for $175 \sim 225 \mathrm{~mJ} / \mathrm{cm}^{2}$.

\section{Results and Discussion}

\subsection{Influence of energy density on the grain mor- phology}

Figure 1 shows the relationship between the Raman intensity and shot number for $175 \sim 225 \mathrm{~mJ} /$ $\mathrm{cm}^{2}$. The crystallinity of poly-Si increases at the critical laser irradiation condition, $225 \mathrm{~mJ} / \mathrm{cm}^{2}$ and 100 shots. Although it is difficult to identify the recovery and primary recrystallization process, the secondary recrystallization is identified as the condition of $225 \mathrm{~mJ} / \mathrm{cm}^{2}$ and 100 shots and 200 shots. Fig. 2(a) and 2(b) show the SEM photographs of the poly-Si surface for the condition of $225 \mathrm{~mJ} / \mathrm{cm}^{2}$ and 32 shots and $225 \mathrm{~mJ} / \mathrm{cm}^{2}$ and 100 shots, respectively. The poly-Si grains become large as increasing the shot number, and the SPC occurs under the low energy density.

Figures 3(a) 3(d) show the SEM photographs of the poly-Si surface for the energy densities of $250 \mathrm{~mJ} / \mathrm{cm}^{2}, 300 \mathrm{~mJ} / \mathrm{cm}^{2}, 350 \mathrm{~mJ} / \mathrm{cm}^{2}$ with the shot numbers of 8 , and the schematic illustration of the SEM photograph of poly-Si surface, respectively. It is found that both the disk-shaped grains and the small size grains by the SPC grow and the area of the disk-shaped grains becomes large as increasing the energy density. It is considered that there is a nucleus in the center of the disk-shaped grains. Although they are observed at the energy density larger than $250 \mathrm{~mJ} / \mathrm{cm}^{2}$ in the present experiment, it is also reported that the disk-shaped grains are formed by the ELA at the energy density of $178 \mathrm{~mJ} / \mathrm{cm}^{2}$ followed by SPC ${ }^{5}$. Fig. 4 shows the relationship between the area ratio of the diskshaped grain and energy density. The area ratio of the disk-shaped grains becomes large as increasing the energy density. Fig. 5 shows the SEM photographs of the poly-Si surface for the condition of

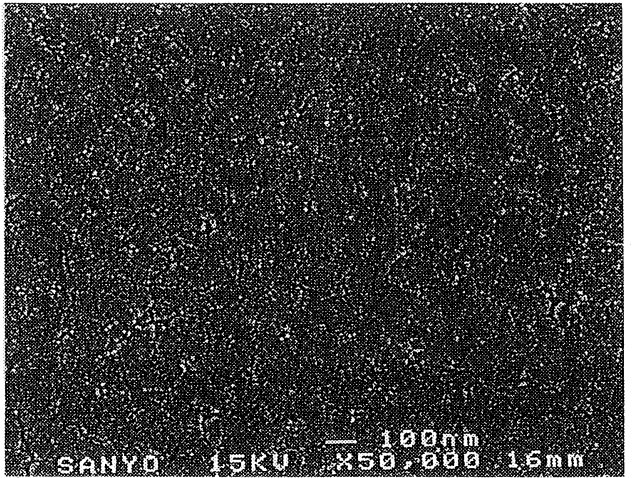

(a)

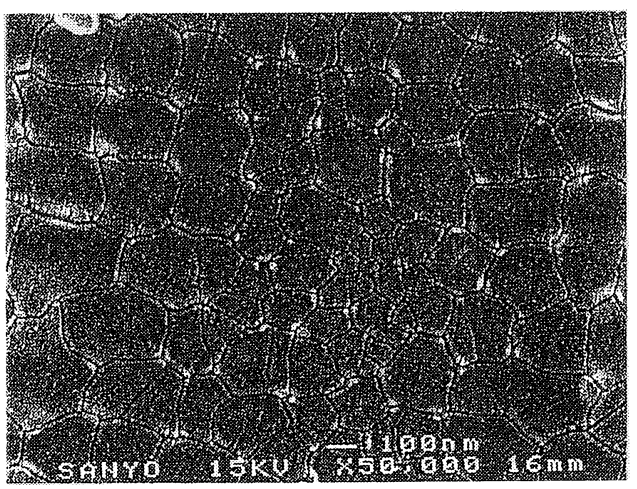

(b)

Fig. 2(a), (b) SEM photographs of the poly-Si surface for the condition of $225 \mathrm{~mJ} / \mathrm{cm}^{2}$ and 32 shots and $225 \mathrm{~mJ} / \mathrm{cm}^{2}$ and 100 shots, respectively.

$400 \mathrm{~mJ} / \mathrm{cm}^{2}$ and 8 shots. It is found that the diskshaped grains are not observed at the large energy density, and the grains with the size of approximately $40 \mathrm{~nm}$ grow.

\subsection{Influence of shot number on the grain mor- phology}

Fig. 6(a) $\sim$ 6(c) show the SEM photographs of the poly-Si surface for the shot numbers of 8,32 and 100 with the energy density of $250 \mathrm{~mJ} / \mathrm{cm}^{2}$, respectively. Fig. 7(a) $\sim$ 7(c) show the SEM photographs of the poly-Si surface for the shot numbers of 8,32 and 100 with the energy density of $300 \mathrm{~mJ} /$ $\mathrm{cm}^{2}$, respectively. Fig. 8(a) $\sim 8($ c) show the SEM photographs of the poly-Si surface for the shot numbers of 8,32 and 100 with the energy density of $350 \mathrm{~mJ} / \mathrm{cm}^{2}$, respectively. It is found that the disk-shaped grains grow and the area of them becomes large as increasing the shot numbers. Although the disk-shaped grains are observed almost entire area for $350 \mathrm{~mJ} / \mathrm{cm}^{2}$, the gatherings of the small grains which are due to the SPC are observed. Fig. 9 shows the relationship between the 


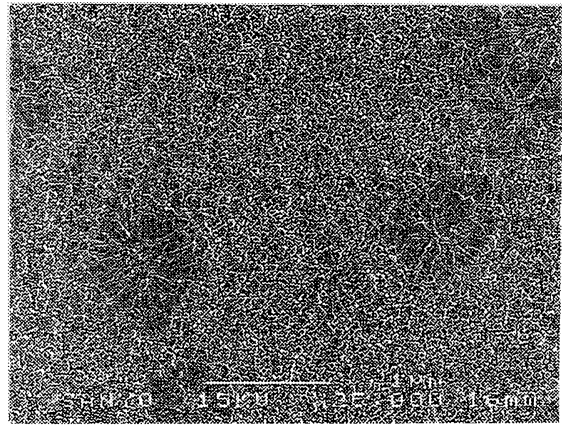

(a)

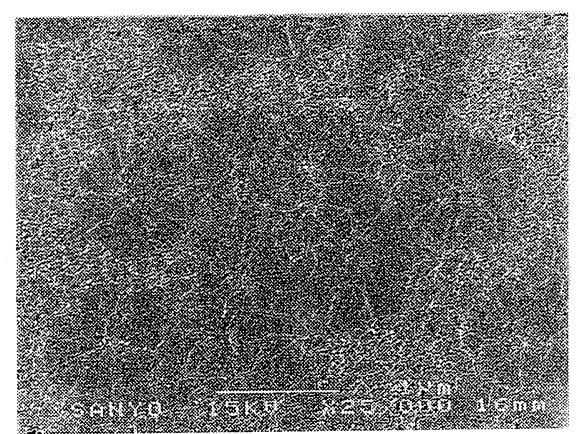

(b)

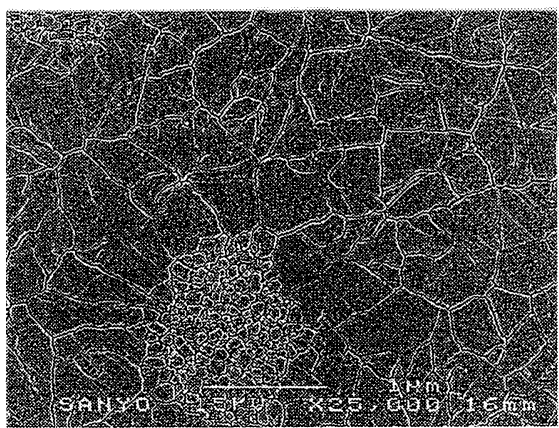

(c)

area ratio of the disk-shaped grain and shot numbers for the energy densities of $250 \sim 350 \mathrm{~mJ} / \mathrm{cm}^{2}$. For $300 \mathrm{~mJ} / \mathrm{cm}^{2}$, the dispersion of the area ratio of the disk-shaped grains is observed. This may be because of the fluctuation of laser energy density at the $\mathrm{Si}$ surface. The area ratio of disk-shaped grains becomes large as increasing the shot numbers at the condition of 250 and $350 \mathrm{~mJ} / \mathrm{cm}^{2}$.

\subsection{Change of the crystal growth mechanism from low to high energy density}

From the experimental results, the change of the crystal growth mechanism dependent on the energy density is discussed. Fig. 10 summarizes the present experimental results. For the low energy density
SPC

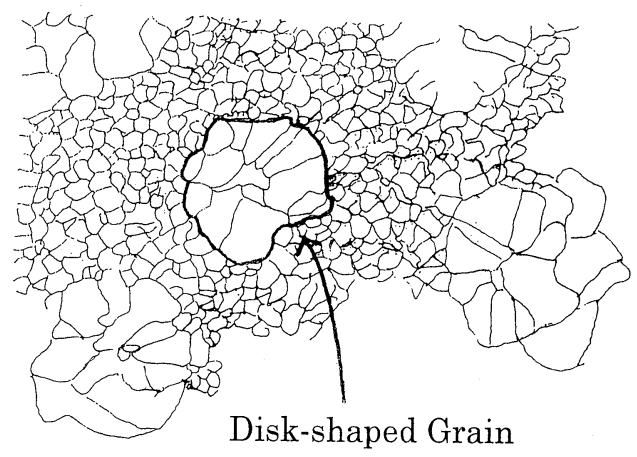

(d)

Fig. 3(a) (d) SEM photographs of the poly-Si surface for the energy densities of $250 \mathrm{~mJ} / \mathrm{cm}^{2}, 300$ $\mathrm{mJ} / \mathrm{cm}^{2}$ and $350 \mathrm{~mJ} / \mathrm{cm}^{2}$ with the shot numbers of 8 , and schematic illustration of the SEM photograph of poly-Si surface, respectively.

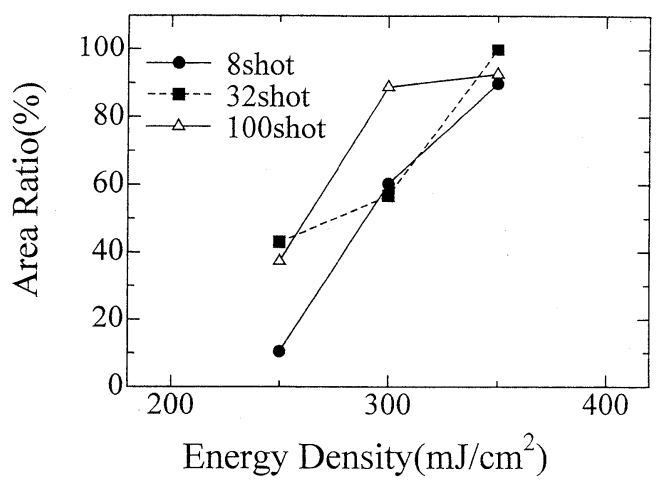

Fig. 4 Relationship between the area ratio of the disk-shaped grain and energy density.

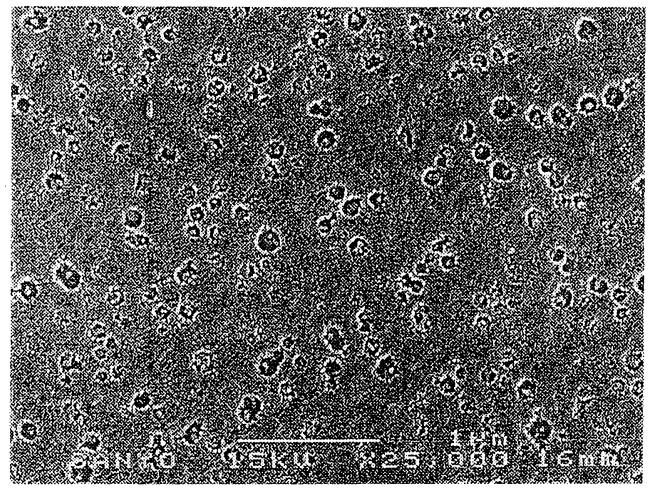

Fig. 5 SEM photographs of the poly-Si surface for the condition of $400 \mathrm{~mJ} / \mathrm{cm}^{2}$ and 8 shots. 


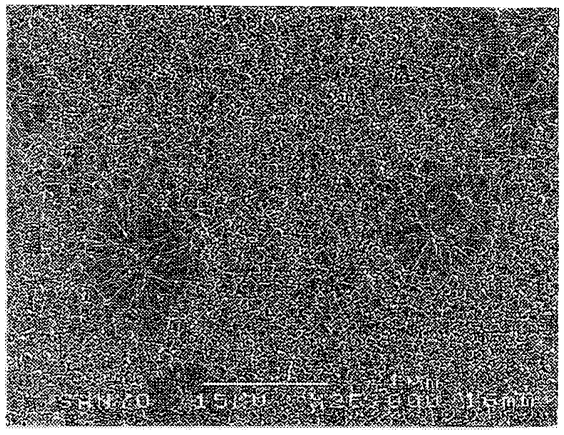

(a)

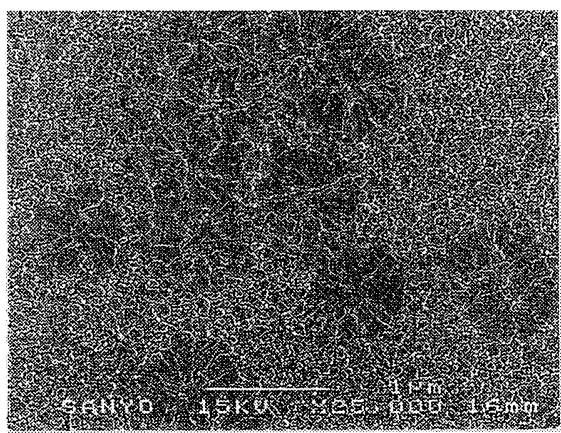

(b)

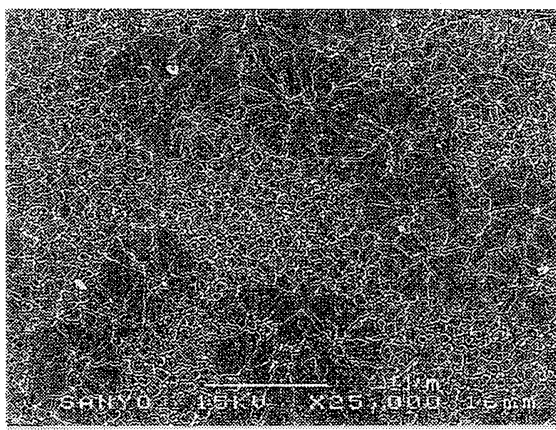

(c)

Fig. 6(a) (c) SEM photographs of the poly-Si surface for the shot numbers of 8,32 and 100 with the energy density of $250 \mathrm{~mJ} / \mathrm{cm}^{2}$, respectively.

smaller than $250 \mathrm{~mJ} / \mathrm{cm}^{2}$, the SPC occurs ${ }^{4}$. For the high energy density larger than $400 \mathrm{~mJ} / \mathrm{cm}^{2}$, crystallization from the super cooled liquid occurs. The conditions of the crystal growth by SPC are painted and those by SCL are not painted. The transient condition between the SPC and the SCL correspond to the area with a tone of color. For a certain energy density, the area ratio by the SCL increases with increasing the shot number and at a

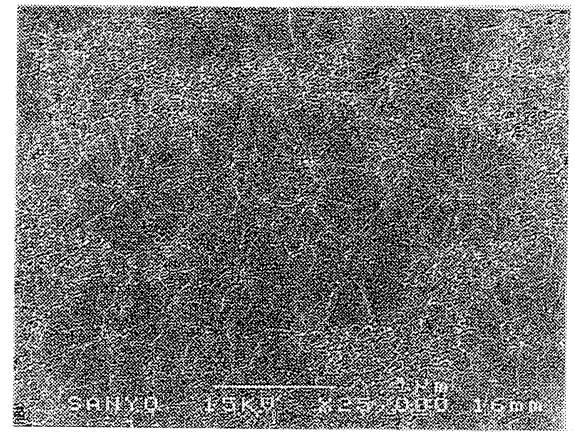

(a)

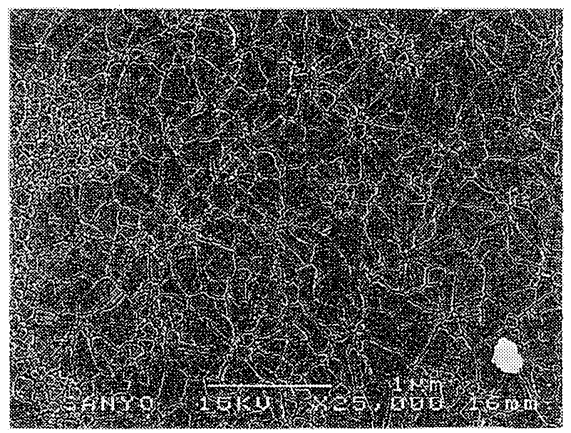

(b)

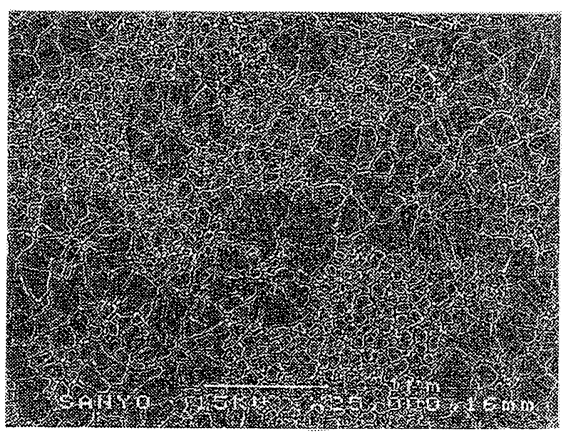

(c)

Fig. 7(a) (c) SEM photographs of the poly-Si surface for the shot numbers of 8,32 and 100 with the energy density of $300 \mathrm{~mJ} / \mathrm{cm}^{2}$, respectively.

certain shot numbers the area ratio equals to 1 . For the shot number, the same phenomenon occurs.

The relationship between the critical grain size $r^{*}$ and the super cooled rate $\Delta T$ is given by eq. (1).

$$
r^{*}=\frac{2 v T_{E}}{L \Delta T}
$$

Here, $v, T_{E}, L$ are the surface free energy per unit area, the melting temperature and the latent heat, 


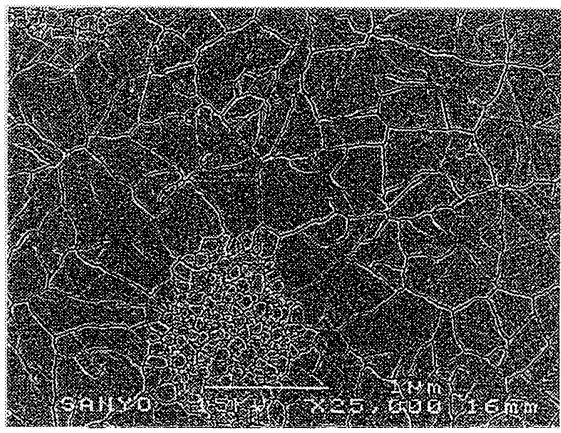

(a)

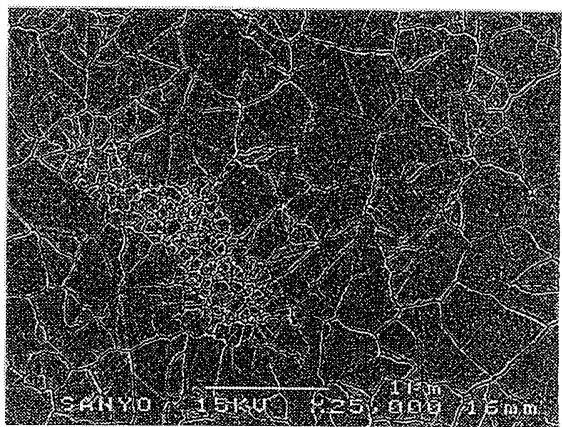

(b)

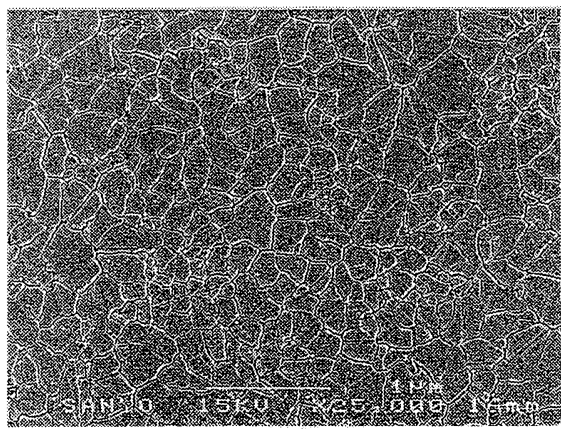

(c)

Fig. 8(a) (c) SEM photographs of the poly-Si surface for the shot numbers of 8,32 and 100 with the energy density of $350 \mathrm{~mJ} / \mathrm{cm}^{2}$, respectively.

respectively. For the high energy density, the super cooled rate $\Delta T$ is large and the critical grain size $r^{*}$ becomes small: The generation of the nucleus is accelerated. This leads to a decrease of the grain size at the energy density of $400 \mathrm{~mJ} / \mathrm{cm}^{2}$. In the transient energy density, the formation of both the disk-shaped grains and the small grains by the SPC occurs. Fig. 11 shows the schematic model of the crystal growth during the transient stage. The dis-

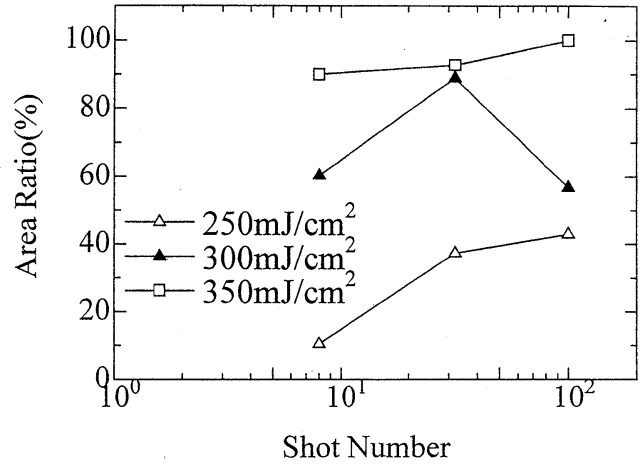

Fig. 9 Relationship between the area ratio of the disk-shaped grain and shot numbers for the energy densities of $250 \sim 350 \mathrm{~mJ} / \mathrm{cm}^{2}$.

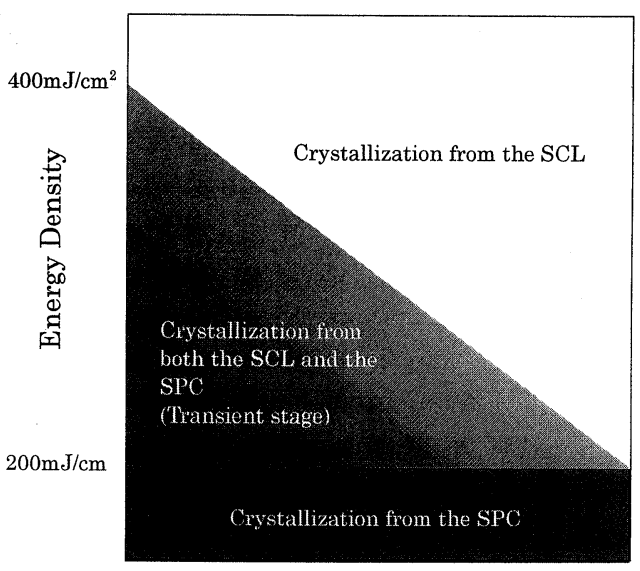

Shot Number

Fig. 10 Summary of the present experimental results.

persion of the surface temperature due to the fluctuation of the energy density during the stage is around the melting temperature of the poly-Si. In the surface area which temperature is higher than the melting temperature, the poly-Si becomes the super cooled liquid and the nuclei of the diskshaped grains are formed in the super cooled liquid. In the surface area which temperature is smaller than the melting temperature, the SPC proceeds. The total energy irradiated to the Si surface is shown by product of the energy density and the shot number. As increasing the total energy, the area ratio of the SCL becomes large due to the increase of the fluctuation. Therefore, the area ratio of the disk-shaped grain becomes large, as increasing the energy density or the shot number. 


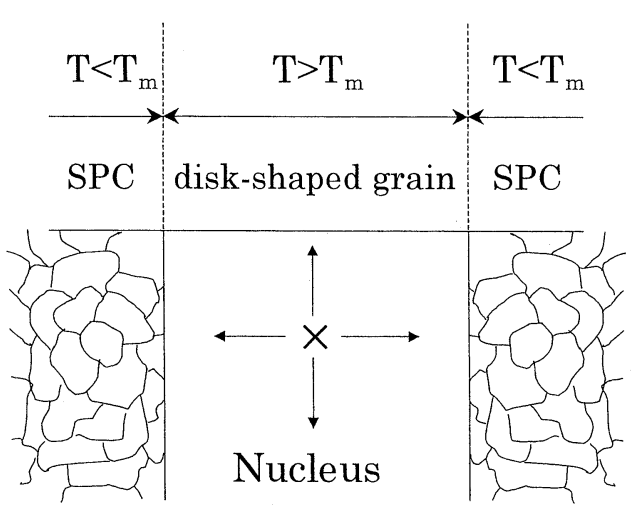

Fig. 11 Schematic model of the crystal growth in the transient area.

\section{Conclusions}

In the range from low to high energy densities, the change of the crystal growth mechanism of recrystallized poly-Si film was examined. In the energy density smaller than $250 \mathrm{~mJ} / \mathrm{cm}^{2}$, the SPC proceeds. In the energy density larger than 400 $\mathrm{mJ} / \mathrm{cm}^{2}$, the crystal growth from the super cooled liquid proceeds. In the transient stage of the energy density from $250 \mathrm{~mJ} / \mathrm{cm}^{2}$ to $400 \mathrm{~mJ} / \mathrm{cm}^{2}$, the dispersion of the surface temperature is around the melting temperature of the poly-Si and both crystal growth mechanisms occur simultaneously: The disk-shaped grains are observed at the melted area and the number of them increases as increasing the energy density and shot number.

\section{Acknowledgements}

The authors would like to thank to Dr. K. Yodoshi, Director of Microelectronics Research Center, SANYO Co. Ltd., for giving us some useful comments at the beginning of the collaboration research. This research is partly supported by "The Akai Foundation" and "The Ube Foundation".

\section{References}

1) N. Matsuo, Y. Aya, N. Kawamoto, F. Anwar, FT. Nouda, H. Hamada and T. Miyoshi: Dig. Tech. Papers. $A M-L C D, 1999$, pp. 95-98.

2) Y. Aya, T. Nouda, H. Abe, Y. Miyai, M. Iseki and H. Hamada: Dig. Tech. Papers. AM-LCD, 1999, pp. 25-28.

3) S. R. Stiffler, P. V. Evans and A. L. Greer: Acta metall. Mater., 40 (1992) 1617.

4) N. Matsuo, Y. Aya, T. Kanamori, T. Nouda, H. Hamada and T. Miyoshi: Jpn. J. Appl. Phys, 39 (2000) 351.

5) Y. Ikeda and T. Noguchi: Proc. $44^{\text {th }}$ Symp. Semiconductor and Integrated Circuit Technolgy, 1993, pp. 187-192. 